\title{
Belastungsanalyse der Dahme durch Sport und Erholung
}

\author{
Prof. Dr. sc. oec. Heinz Kroske, Dr. oec. Bernd Lehne
}

Von der Senatsverwaltung für Stadtentwicklung, Umweltschutz und Technologie wurde an die Technische Fachhochschule Wildau ein Forschungsauftrag zum o. g. Thema vergeben. Die nachfolgenden Ausführungen stellen einige Aspekte der in zwei Teilen vorliegenden wissenschaftlichen Studie dar.

\section{Aufgabenstellung}

Die Studie stellt sich das Ziel, Handlungsempfehlungen für Behörden zu erarbeiten, die dazu beitragen, eine konfliktminimierte Mehrfachnutzung im Revier Dahme zu erreichen. Die durchzuführenden Untersuchungen erstrecken sich auf die Erfassung von Wassersport und gewerblichem Schiffahrtsverkehr auf der Dahme von der Spindlersfelder Eisenbahnbrücke bis Rauchfangswerder Nord (Untersuchungsgebiet) sowie auf die Verkehrsbelastungen durch den ÖPNV und den privaten Verkehr im Uferbereich der Dahme.

Die Handlungsempfehlungen basieren auf Untersuchungsergebnissen und Bewertungen die eine differenzierte verkehrliche Belastung des Gewässers und seiner Uferbereiche nachweisen.

Durch unterschiedliche Nutzungsanspriiche treten Konflikte u.a. im Bereich der Uferzone auf, die durch entsprechende Maßnahmen zu steuern sind. Die Konflikterfassung im Uferbereich gehört zum Schwerpunkt der Untersuchung. Konflikte mit dem Natur- und Gewässerschutz treten vor allem im Bereich von Badestellen, besonders an freien Badestellen, durch Beeinträchtigung der Uferzonen durch Badende, Boote, Surfer und den ruhenden Verkehr auf.

Zu erfassen sind die Frequentierung der Badestellen und der Naherholungszentren, die Art des Erreichens der Erholungszentren (ÖPNV, private Pkw, mit dem Rad, zu Fuß), die Belastung der Uferzone und der Bootsverkehr, der die Zentren anläuft und verläßt.

Zur Belastung tragen auch Bootshäfen, -stege, Anlagestellen u.a. bei. Daher muß der Bootsverkehr an diesen Zentren erfaßt werden

Herauszuarbeiten sind konfliktträchtige und übernutzte Uferbereiche sowie Verdichtungsabschnitte auf dem Gewässer. Für diese Konfliktbereiche sind Handlungsempfehlungen zu erarbeiten, damit sich die Konzentration von Wassersport an infrastrukturell und ökologisch geeigneten Bereichen vollziehen kann.

Die hohe ökologische Qualität der Uferbereiche der Dahme, die natürlichen Landschaftselemente (Auwaldstreifen, Inseln, Buchten) darf durch Übernutzung nicht gemindert werden.

Durch repräsentative Erfassungen an sieben Erfassungspunkten sowie zu unterschiedlichen Tages- und Jahreszeiten (Schwerpunkt ist der Saisonbetrieb Mai - Oktober) werk- und sonntags sowie feiertags sind die erfor- derlichen Daten zur Auswertung und Bewertung zu statistisch repräsentativen Aussagen zu gewinnen. Basierend auf der Auswertung vorhandener Unterlagen und Informationen sowie auf der Auswertung eigener Untersuchungen, werden die Ergebnisse nach ihrer Umweltverträglichkeit oder -unverträglichkeit eingeschätzt und eine Bewertung der verkehrlichen Belastung der Uferbereiche vorgenommen.

Die Spezifik des Untersuchungsgebietes wird durch Begehungen erfaßt, besonders an den Punkten, an denen es voraussehbar durch ein hohes Verkehrsaufkommen zu starken Belastungen während der Saison kommt (Langer See, Zeuthener See).

Erfassungslisten zur Ermittlung der verschiedenartigen Verkehrsströme wurden entwickelt und eingesetzt.

Die Ergebnisse der Erfassung werden auf Karten im Maßstab 1: 10000 und die Richtungen des Schiffsverkehrs an Schwerpunkten des Untersuchungsgebietes auf vergrößerten Kartenausschnitten dargestellt.

\section{Charakterisierung des Untersuchungs- gebietes}

Das Revier der Dahme (Untersuchungsgebiet) umfaßt Teile der Spree - Oder - Wasserstraße, einschließlich der Großen Krampe und des Seddinsees sowie Teile der DahmeWasserstraße. Es hat eine Länge von insgesamt 20,7 km. Es reicht von der Einmündung der Müggelspree an der Dammbrüicke in Köpenick, km 32,8 der Spree - Oder Wasserstraße, einschließlich des Langen Sees, der Großen Krampe und des Seddinsees, bis km 45,10, Beginn des Oder - Spree - Kanals. Die zum Untersuchungsgebiet gehörende Dahme-Wasserstraße beginnt mit dem $\mathrm{Km} \mathrm{0,0}$ an der Schmöckwitzer Brücke und endet beim $\mathrm{km} 4$ bei Rauchfangswerder.

\begin{tabular}{|l|l|l|l|}
\hline Wasserstraße & Bezeichnung & Länge & Zugehörigkeit \\
\hline \multirow{2}{*}{$\begin{array}{l}\text { Oder - Spree - } \\
\text { Wasserstraße }\end{array}$} & Dahme & $\begin{array}{l}\text { von } \mathrm{km} 32,8- \\
37,4(4,6 \mathrm{~km})\end{array}$ & Berlin \\
\cline { 2 - 4 } & Langer See & $\begin{array}{l}\text { von } \mathrm{km} 37,4- \\
43,5(3,0 \mathrm{~km})\end{array}$ & Berlin \\
\cline { 2 - 4 } & Große Kramoe & $\begin{array}{l}\text { von } \mathrm{km} 0,0- \\
3,0(3,0 \mathrm{~km})\end{array}$ & Berlin \\
\cline { 2 - 4 } & Seddinsee & $\begin{array}{l}\text { von } \mathrm{km} 0,0- \\
3,0(3,0 \mathrm{~km})\end{array}$ & $\begin{array}{l}\text { Berlin/ } \\
\text { Brandenburg }\end{array}$ \\
\hline Dahme Wasserstraße & Zeuthener See & von km $0,0-$ & Berlin/ \\
& & $4,0(4,0 \mathrm{~km})$ & Brandenburg \\
\hline
\end{tabular}

Gesamt:

$20.7 \mathrm{~km}$

Tabelle 1: Bestand und Länge des Untersuchungsgebietes

Der gegenwärtige Bootsbestand im Untersuchungsgebiet beläuft sich auf eine Anzahl von ca. 3500 Sport- 
booten. Durch Befragung der im Revier ansässigen Vereine, Bootshäuser und Bootsvermietungen wurden tatsächlich erfaßt 3035 Boote. Da von den 71 Befragten jedoch nur 47 Angaben machten und desweiteren sich auch Sportboote auf den Grundstuicken privater Anlieger befinden, muß tatsächlich von einem höheren Bootsbestand im Revier (ca. 3.500) ausgegangen werden, als in der nachfolgenden Tabelle ausgewiesen.

\begin{tabular}{|l|l|l|l|l|l|l|}
\hline & & Motorboote & Ruderboote & Segelboote & Kanuboote & Gesamt \\
\hline Wasserstrake & Dahme & 108 & 238 & 252 11 & 55 & 653 \\
& $\begin{array}{l}\text { L.anger See } \\
\text { dar. GroBe } \\
\text { Krampe }\end{array}$ & 90 & 461 & 405 & 239 & 1338 \\
& $\begin{array}{l}\text { Seddinsee } \\
\text { Zeuthener } \\
\text { See }\end{array}$ & 586 & 1 & 50 & 18 & 159 \\
\hline Gesamt & & 17 & 223 & 144 & 770 \\
\hline
\end{tabular}

1) davon etwa 40 s mit eigenem Motor

Tabelle 2: Erfaßter Bootsbestand im Revier der Dahme

Vom ermittelten Bootsbestand ausgehend ergibt sich folgende Rangfolge:

$\begin{array}{lll}\text { 1. Segelboote } & 997 & 32,85 \% \\ \text { 2. Motorboote } & 783 & 25,80 \% \\ \text { 3. Ruderboote } & 754 & 24,84 \% \\ \text { 4. Kanuboote } & 501 & 16,51 \%\end{array}$

Aus den ermittelten Bestandsdaten und im Vergleich mit einer 1994 durchgefuihrten ABM-Studie ergeben sich folgende Schlußfolgerungen:

1. Der Bootsbestand hat sich in den Jahren 1994- 1997 quantitativ nicht wesentlich verändert. Eine steigende Tendenz zum Betreiben von größeren Kajütmotorbooten ist vorhanden.

2. Die Entwicklung des Bootsbestandes wurde 1994 stärker eingeschätzt als 1997.

3. Hinsichtlich der Steganlagen besteht kein nennenswerter Erweiterungsbedarf.

4. Die Mitgliederentwicklung in den Sportvereinen hat sich quantitativ nicht wesentlich verändert und wurde 1994 noch stärker als 1997 eingeschätzt.

Das Untersuchungsgebiet ist im oberen Verlauf der Dahme, von Köpenick bis Grünau mit Wohn-, Industrieund Gewerbegebieten sowie mit Sportanlagen beiderseitig bebaut. Am Langen See sind im südwestlichen Teil die Ortsteile Karolinenhof und Schmöckwitz als geschlossene Siedlungen vorhanden sowie am Zeuthener See, Südostufer die Siedlung Rauchfangswerder. Das gesamte Ostufer des Sees ist durch die Gemeinden Eichwalde und Zeuthen bebaut. Am nördlichen Ende der Großen Krampe liegt der Ortsteil Müggelheim und an der nordöstlichen Seite des Seddinsees die Gemeinde Gosen, sowie weiter südlich die Siedlung Zwiebusch. Alle anderen Bereiche des Untersuchungsgebietes sind unbebaut und als weiträumige und zusammenhängende Erholungsgebiete genutzt. In ihnen sind alle Arten von Wasser- und Freizeitsport, Wandern, Radfahren und Camping vorherrschende Nutzungen, besonders während der Sommermonate.
Die Nord- und die Südufer des Langen Sees, die Ost- und die Westufer der Großen Krampe, sowie die Ost- und die Westufer des Seddinsees und das Ostufer des Zeuthener Sees gehören dazu.

Die die Ufer säumenden Forsten (vorwiegend Kiefernund Mischwaldbestände) werden als Erholungswald genutzt und entwickelt. Mehr als 2/3 des gesamten Untersuchungsgebietes gehören zu den Erholungsgebieten, die schon jahrzehntelang von den Berlinern und Brandenburgern sowie zahlreichen Besuchern für Sport und Erholung genutzt werden.

Diese Gebiete in ihrer Funktion zu erhalten und zu stabilisieren ist langfristiges Ziel des Berliner und Brandenburger Naturschutzes.

Ingesamt besitzen die Erholungsgebiete im Untersuchungsbereich einen hohen Schutzstatus durch den Natur-, Landschafts- und Trinkwasserschutz. Größere Naturschutzgebiete befinden sich im nordöstlichen Teil des Seddinsees, die Gosener Wiesen und westlich des Langen Sees, das Feuchtgebiet der Krummen Lake.

Das Entwicklungsziel für den Landschaftsraum einschließlich der Ortsteile muß ein konfliktarmes Nebeneinander aller Nutzungsansprüche gewährleisten und die Anforderungen an den Trinkwasserschutz, Schutz des Landschaftscharakters, Biotop- und Artenschutz mit der baulichen Entwicklung sowie mit der Nutzung für Sport und Erholung in Übereinstimmung bringen.

\section{Analyse der wasser- und landseitigen Nutzung}

Um die Ziele der Studie zu erreichen wurden in Abstimmung mit dem Auftraggeber sieben Erfassungspunkte (EP 1 - 7) festgelegt.

EP 1 Rauchfangswerder, Fährallee

EP 2 Schmöckwitzer Brücke, südliche Brückenseite (Richtung Zeuthener See)

EP 3 Karolinenhof -Richtershorn

EP 4 Seddinsee, nördlicher Teil Gosener Kanal und südöstlicher Teil Oder-Spree-Kanal

EP 5 Langer See, Grünau (Strandbad) bis Bammelecke EP 6 Dahme/Köpenick Kleingartenanlage

EP 7 Köpenick, Dammbrücke

Die Erfassungspunkte wurden so gewählt, daß eine vollständige Erfassung des gesamten Bootsverkehrs auf der Dahme möglich war. Die Zählungen bezogen sich auf dem Zeitraum Mai bis Oktober 1996.

Wesentliche Erfassungskriterien waren:

- Nutzungsarten wie - Fahrgastschiffahrt(FB), gewerbliche Schiffahrt(GB), Motorboote $(\mathrm{MB})$, Sportboote (SP), Kleinsportboote (KSB), Segelboote (SEG), Surfer (SUR). Badende (BAD), Sonstige (SON)

- Verkehrsarten

- Richtungsverkehr von und nach Berlin

- Richtungsverkehr an vier Schwerpunkten (EP 1, EP $4(2 x)$ und EP 7)

- Quellverkehr am Erfassungspunkt 
- Einzelboote und Schubverband (als weitere Untergliederung der gewerblichen Schiffahrt)

- sonstige Kriterien

- Wochentag

- Uhrzeit

- Wetter (Temperatur, Wettereinfluß)

Um die Ergebnisse zwischen den verschiedenen Erfassungspunkten und den unterschiedlichen Erfassungszeiten vergleichbar zu machen, wurden sie auf einen Stundenwert dividiert und für jeden Erfassungspunkt und die Nutzungsart ein Durchschnitt gebildet, der als Dichtefaktor bezeichnet wird.

Auf der Grundlage der im Revier erfaßten Nutzungsarten ergibt sich hinsichtlich Dichtefaktor und Höchstbelastungen folgender Überblick:

\begin{tabular}{|c|c|c|c|c|c|c|c|c|c|}
\hline $\begin{array}{l}\text { Nut- } \\
\text { zungs- } \\
\text { art }\end{array}$ & $\mathrm{FB}$ & GB & MB & $\mathrm{SP}$ & KSP & SEG & SUR & $\mathrm{BAD}$ & SON \\
\hline $\begin{array}{l}\text { Dichte- } \\
\text { faktor/h }\end{array}$ & 1,15 & 1.41 & 20,15 & 2,03 & 3,77 & 3,98 & 0.25 & 4,06 & 0,26 \\
\hline $\begin{array}{l}\text { höchste } \\
\text { Bela- } \\
\text { stung }\end{array}$ & 7,50 & 7,50 & 87,0 & 18,50 & 46,00 & 51,50 & 20,00 & 157,50 & 3,00 \\
\hline $\begin{array}{l}\text { Erfas- } \\
\text { sungs- } \\
\text { punkt }\end{array}$ & 7 & 6 & $2 \pi$ & 7 & 2 & 5 & 5 & 3 & 6 \\
\hline Datum & 21.07 & 15.05. & $\begin{array}{r}27.07 \\
17.08\end{array}$ & 21.08 & 21.09 & 12.10 & 12.10 & 08.06 & 15.05 . \\
\hline $\begin{array}{l}\text { Wo- } \\
\text { chentag }\end{array}$ & So & $\mathrm{Mi}$ & Sa/So & Mi & Sa & Sa & Sa & Sa & Mi \\
\hline $\begin{array}{l}\text { Tages- } \\
\text { tempe- } \\
\text { ratur }\end{array}$ & $25^{\circ} \mathrm{C}$ & $16^{\circ} \mathrm{C}$ & $\begin{array}{l}23^{\circ} \% \\
25^{\circ} \mathrm{C}\end{array}$ & $31^{\circ} \mathrm{C}$ & $14^{\circ} \mathrm{C}$ & $13^{\circ} \mathrm{C}$ & $13^{\circ} \mathrm{C}$ & $31^{\circ} \mathrm{C}$ & $16^{\circ} \mathrm{C}$ \\
\hline Zeit & $\begin{array}{l}16.00- \\
18.00\end{array}$ & $\begin{array}{l}16.00- \\
18.00\end{array}$ & $\begin{array}{l}14.00 \\
16.00\end{array}$ & $\begin{array}{l}16.00 \\
18.00\end{array}$ & $\begin{array}{l}14.00- \\
16.00\end{array}$ & $\begin{array}{l}10.00- \\
12.00\end{array}$ & $\begin{array}{l}10.00 \\
12.00\end{array}$ & $\begin{array}{l}14.00 \\
16.00\end{array}$ & $\begin{array}{l}16.00- \\
18.00\end{array}$ \\
\hline
\end{tabular}

Tabelle 3: Dichtefaktor und Höchstbelastungen

Die Analyse des Dichtefaktors für die unterschiedlichen Boots -Nutzungsarten zeigt eine Reihenfolge, die durch die Motorboote angefuihrt wird.

1. Motorboote

2. Segler

3. Kleinsportboote

4. Sportboote

5. gewerbliche Boote

6. Fahrgastboote

7. sonstige Boote

8. Surfer

Die Motorboote sind sowohl von der Größenordnung als auch von den Belastungen wie Lärm, Verschmutzung der Gewässer durch Öl und Treibstoff, ökologische Schäden (Beeinträchtigung des Röhrichts, Uferauswaschungen und Unterspülen unbefestigter Ufer, Belastung von Pflanzen, Tieren und Menschen durch Abgase und Lärm) die Bootsart, von der die größte ökologische Belastung im Revier Dahme ausgeht.

Die Segler, die etwa $40 \%$ motorgetrieben ins Segelrevier gefahren werden, sind unter diesem Aspekt analog ein- zuschätzen. Allerdings gemindert, weil sie auch bei Motorbetrieb nicht die Geschwindigkeit erreichen und nicht die Strecken fahren, wie die Motorboote.

Die gewerbliche und Fahrgastschiffahrt, an 5. und 6 . Stelle liegend, sind jedoch von ihrer Zahl so gering, daß sie als Belastungsfaktor zwar die gleichen und wegen ihrer Größe (Motorstärke) höhere Wirkungen wie die Motorboote aufweisen, jedoch mit einer oder eineinhalb Einheiten pro Stunde eine sehr geringe Dichte aufweisen.

Die Analyse der Höchstbelastungen, d.h. der größten Anzahl von Bootseinheiten, die im Untersuchungsgebiet und im Untersuchungszeitraum gezählt wurden, zeigt nahezu die gleiche Reihenfolge, wie die Analyse des Dichtefaktors. Die Ausnahme bilden hier die Surfer, die auf Platz 4 vorrüicken.

Differenziert sind jedoch die Werte der jeweiligen Höchstzählungen. Jeweils drei bis zwei Höchstbelastungen treten am

- Erfassungspunkt 7 (Motorboote, Sportboote und Fahrgastboote)

- Erfassungspunkt 6 (gewerbliche Boote und sonstige)

- Erfassungspunkt 5 (Segler und Surfer)

- Erfassungspunkt 2 (Motorboote und Kleinsportboote) sowie am

- Erfassungspunkt 3 (Badende) auf.

Was die Daten, Wochentage, Temperatur und Uhrzeit betrifft, so gibt es hier eine große Streuung bei den Daten von Mai bis Oktober, obwohl gerade die Zähltage im Mai und Oktober relativ kühl waren $\left(16^{\circ} \mathrm{C}\right.$ und $\left.13^{\circ} \mathrm{C}\right)$. Die Wochenenden zeigen sich in den überwiegenden Fällen als Höchstbelastungstage, besonders wenn dazu noch hochsommerliche Temperaturen auftreten. Die Zeit von $14.00-18.00 \mathrm{Uhr}$ erweist sich als Höchstbelastungszeit.

Auch bei den einzelnen Erfassungspunkten erweisen sich die Motorboote als jene Nutzungsart, die mit Abstand die höchste Dichte aufweist. Die höchsten Werte treten an den Erfassungspunkten vier, zwei und sechs auf.

Relativ gleichmäßig über alle Erfassungspunkte verteilt sind die Nutzungsarten Fahrgastschiffahrt und gewerblicher Verkehr.

Schwerpunkte an einzelnen Erfassungspunkten bilden:

$\begin{array}{ll}\text { Badende } & \text { EP3 } \\ \text { Segler } & \text { EP4 } \\ \text { Surfer } & \text { EP5 (aber insgesamt gering) } \\ \text { Kleinsportboote } & \text { EP5 } \\ \text { Sportboote } & \text { EP5 }\end{array}$

\section{Ergebnisse der Analyse}

Wie aus der Übersicht über die Erfassungspunkte ersichtlich, wurde die Erfassung der wasser- und landseitigen Nutzung des Untersuchungsgebietes an sieben Erfassungspunkten vorgenommen. Um relevante Aussagen über die spezifischen Belastungen in unterschiedlichen Bereichen zu erhalten und daraus konkrete Hand- 


\begin{tabular}{|c|c|c|c|c|c|c|c|c|c|c|c|c|}
\hline Bereich & EP & MB & SEG & $\begin{array}{l}\mathrm{SB} / \\
\mathrm{KSB}\end{array}$ & GB & FB & BAD & Wand. & Radf. & ruhender Verkehr & Durchgangsverkehr & $\begin{array}{l}\text { Zelt- } \\
\text { plät- } \\
\text { ze } \\
\end{array}$ \\
\hline \multirow{3}{*}{$\begin{array}{l}\text { 1.Grünauer Erho- } \\
\text { lungsgebiet }\end{array}$} & 3 & 28 & 31 & 24 & 3 & 2 & 315 & 161 & 218 & 22 & \multirow{2}{*}{$\begin{array}{l}\text { Tram } \\
68 \\
\end{array}$} & 0 \\
\hline & 5 & 27 & 12 & 38 & 4 & 1 & 48 & 160 & 200 & 16 & & 0 \\
\hline & ges. & 55 & 43 & 62 & 7 & 3 & 363 & 321 & 418 & 38 & & 0 \\
\hline \multirow{3}{*}{$\begin{array}{l}\text { 2. Wendenschloß } \\
\text { Krampenburg } \\
\text { Müggelheim }\end{array}$} & 3 & & & & & & & & & & & \\
\hline & 5 & & & & & & & & & & 0 & 2 \\
\hline & ges. & 55 & 43 & 62 & 7 & 3 & 220 & 21 & 35 & 250 & 0 & 2 \\
\hline \multirow{3}{*}{$\begin{array}{l}\text { 3.Schmöckwitzer Br. } \\
\text { Zeuthener See } \\
\text { Rauchfangswerder }\end{array}$} & 2 & 87 & 2 & 51 & 3 & 2 & & 0 & 0 & 333 & \multirow{2}{*}{$\begin{array}{l}\text { Schmöckwitz-Werns- } \\
\text { d. } 10000 \mathrm{Fahrz} / \text { tägl. }\end{array}$} & 2 \\
\hline & 1 & 38 & 7 & 7 & 1 & 2 & $400^{21}$ & 25 & 15 & 270 & & 0 \\
\hline & ges. & 125 & 9 & 58 & 4 & 4 & 400 & 25 & 15 & 603 & 4166 & 2 \\
\hline \multirow[t]{3}{*}{ 4.Seddinsee } & $44_{1}$ & 36 & 71 & 7 & 7 & 4 & 30 & 83 & 15 & 20 & \multirow{3}{*}{$\begin{array}{l}0 \\
\text { Bus } 169\end{array}$} & 0 \\
\hline & $44_{2}$ & 40 & 23 & \begin{tabular}{|l|}
9 \\
\end{tabular} & 0 & 1 & 0 & 16 & 3 & 51 & & 1 \\
\hline & ges & 76 & 94 & 16 & 7 & 5 & 30 & 99 & 18 & 71 & & 1 \\
\hline \multirow[t]{3}{*}{ 5. Nördliche Dahme } & 6 & 71 & 35 & 26 & 7 & 2 & 6 & 0 & 0 & \multirow{3}{*}{$\begin{array}{l}\text { in Wohnbereichen } \\
\text { keine Zahlen }\end{array}$} & \multirow{3}{*}{$\begin{array}{l}\text { Wendenschloßstr. } \\
\text { Lange Br.,Grünauer-, } \\
\text { Regattastr.,Dammbr. }\end{array}$} & 0 \\
\hline & 7 & 87 & 2 & 26 & 3 & 7 & 0 & 0 & 0 & & & 0 \\
\hline & ges. & 158 & 37 & 52 & 10 & 9 & 6 & 0 & 0 & & & 0 \\
\hline
\end{tabular}

1) Durch die Addition von Sport- und Kleinsportbooten wegen ihrer gleichen ökologischen Wirkungen sind die Werte hơher als in anderen Teilen der Studie. 2) Badestelle Schmöckwitzer Brücke

41) Oder-Sprec-Kanal

42) Gosener Kanal

Tabelle 4: Wasser- und landseitige Belastung von Teilberecihen des Untersuchungsgebietes (auf Basis erfaßter Höchstwertw/h an den EP)

lungsempfehlungen abzuleiten, wird das Untersuchungsgebiet in fünf Bereiche gegliedert:

1. Grünauer Naherholungsgebiet 1)

2. Wendenschloß - Krampenburg - Müggelheim ${ }^{1)}$

3. Schmöckwitzer Brïcke - Zeuthener See - Rauchfangswerder

4. Seddinsee

5. Nördliche Dahme

Diesen Bereichen werden die Erfassungsergebnisse von jeweils zwei Erfassungspunkten (EP) und die Erfassung landseitiger Belastungen zugeordnet. Die in der Tabelle 4 erscheinenden Werte sind die jeweils an den Erfassungspunkten erfaßten Höchstwerte je Stunde. Diese Daten werden dann in einer Belastungsmatrix bewertet und enthalten die Aussagen über wasserseitige Verdichtungsabschnitte und landseitige Nutzungsschwerpunkte (vgl. Tabelle 5).

Aus der Belastungsmatrix (Tabelle 5) ergibt sich eine differenzierte wasser- und landseitige Bewertung des Untersuchungsgebietes. Die Verdichtungsabschnitte auf den Gewässern sowie die landseitigen Nutzungsschwerpunkte werden ersichtlich.

Der Bereich mit der höchsten Belastung ist der des Grünauer Naherholungsgebietes, gefolgt vom Zeuthener See, der nördlichen Dahme, das Wendenschloßufer (Nordufer) des Langen Sees und den Seddinsee.

Insgesamt muß festgestellt werden, daß der Lange See an beiden Ufern hohe wasser- wie landseitige Belastungen aufweist (mit Differenzierungen zwischen Grünauer- und Wendenschloßufer) und sich dieser Bereich als das am stärksten durch Wassersport und Freizeitnutzung belastete Erholungsgebiet im Untersuchungsgebiet heraushebt.

Das Grünauer Naherholungsgebiet hat wasserseitig starke Belastungen durch Motorboote und wasser-wie landseitig durch die Regattastrecke, sowie durch Badende in einem öffentlichen Bad und zahlreiche freie Bade- stellen. Mittelstarke Belastungen gehen von den Wanderern und Radfahrern aus, die hier die höchsten Belastungswerte erreichen.

Während man im Bereich Wendenschloß, Krampenburg, Müggelheim wasserseitig etwa die gleichen Belastungen wie dem Grünauer Naherholungsgebiet zurechnen muß, sind die landseitigen Belastungen dieses Gebietes nur etwa halb so groß wie die wasserseitigen.

Ähnlich trifft diese Einschätzung für den Seddinsee zu. Hier sind wasserseitig Segler und Motorboote hervorzuheben, während landseitig weitaus geringere Belastungen vorliegen.

Der Bereich der nördlichen Dahme hat ebenfalls höhere wasserseitige, denn landseitige Belastungen.

Im Bereich des Zeuthener Sees, besonders an der Schmöckwitzer Brücke (Nordteil des Sees) und bis Rauchfangswerder ist die wasser- und landseitige Belastung etwa gleich hoch. Das hat seine Ursache in der vom ruhenden und Durchgangsverkehr ausgehenden Belastungen im nördlichen Teil des Bereiches (Schmöckwitzer Brïcke) und in den Belastungen, die von den Zeltplätzen ausgehen.

Wasserseitig treten hier sehr hohe Belastungen durch Motorboote, die gewerbliche- und Fahrgastschiffahrt sowie durch Badende auf.

Das westliche Ufer des Zeuthener Sees ist fast durchgängig bebaut. Belastungen durch Wanderer und Radfahrer treten hier nicht auf. Die Belastung durch Badende ist gering. Nur die Anlieger und der ruhende Bootsverkehr treten hier als geringe Belastungsfaktoren auf.

Im Ergebnis der Analyse können wasserseitig die Regattastrecke im Langen See, der nördliche Teil des Zeuthener Sees im Bereich der Schmöckwitzer Brücke sowie der Seddinsee im Bereich des Oder-Spree- und des Gosener Kanals als Verdichtungsabschnitte der Gewässer bezeichnet werden.

Eine besondere Rolle nimmt die nördliche Dahme im Bereich Dammbrïcke ein, da sich hier der wasserseitige 


\begin{tabular}{|c|c|c|c|c|c|c|c|c|}
\hline \multirow{2}{*}{$\begin{array}{l}\text { Bereich des } \\
\text { Untersuchungsge- } \\
\text { bietes }\end{array}$} & \multicolumn{6}{|c|}{ Belastungen } & \multicolumn{2}{|r|}{ Gesamteinschätzung } \\
\hline & $\begin{array}{l}\text { Höchst- } \\
\text { werte/h }\end{array}$ & $\begin{array}{l}\text { wasserseitig } \\
\text { Bewertung }\end{array}$ & Pkt. & $\begin{array}{l}\text { Höchst- } \\
\text { werte/h }\end{array}$ & $\begin{array}{l}\text { landseitig } \\
\text { Bewertung }\end{array}$ & Pkt. & Pkt & verbale Kurzcharakteristik \\
\hline $\begin{array}{l}\text { 1.Grünauer Naher- } \\
\text { holungsgebiet }\end{array}$ & \begin{tabular}{|lr} 
MB & 55 \\
SEG & 43 \\
& \\
SB/ & \\
KSB & 62 \\
GB & 7 \\
& \\
FB & 3 \\
Bad & 363 \\
Strandbad \\
Grünau \\
4rú \\
Regatta- \\
strecke \\
120 \\
\end{tabular} & 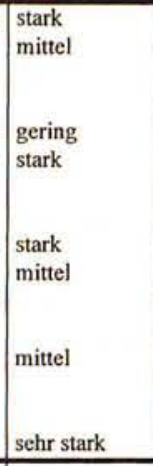 & $\begin{array}{l}15 \\
70 \\
\\
51 \\
45\end{array}$ & $\begin{array}{l}\text { Wand. } 321 \\
\text { Radf. } 418 \\
\\
\text { ruhender } \\
\text { Verkehr. } 38 \\
\text { Durch. } \\
\text { Verkehr } \\
\text { Tram } 68 \\
\text { Zeltplatze } \\
\text { Regattastr. } \\
\text { bis } \\
500 \\
\text { Besucher }\end{array}$ & \begin{tabular}{|l}
$\begin{array}{l}\text { mittel } \\
\text { mittel }\end{array}$ \\
gering \\
mittel \\
nicht relevant
\end{tabular} & $\begin{array}{l}30 \\
30\end{array}$ & & $\begin{array}{l}\text { Traditionelles Naherholungsgebiet mit starkem Boots- und Besu- } \\
\text { cherverkehr, besonders bei Veranstaltungen an der } \\
\text { Regattastrecke. } \\
\text { Bei Veranstaltungen nehmen bis } 100 \text { Motorboote und bis zu } \\
5000 \text { Besucher teil. Die meisten Veranstaltungen finden mit Ka- } \\
\text { nu- und Ruderboten statt. Aber auch starke touristische Nut- } \\
\text { zung, kaum Belastung durch ruhenden Verkehr, da in Wasser- } \\
\text { năhe keine nennenswerten Parkmóglichkeiten vorhanden, Strand- } \\
\text { bad Grünau und Badestellen an der Bammelecke vorhanden } \\
\text { Das Strandbad Grunau hatte } 1996: 1995 \text { einen } \\
\text { Besucherrückgang auf } 1 / 3(20.000: 62.000) \text {. }\end{array}$ \\
\hline \begin{tabular}{|l} 
Punkte gesamt \\
\end{tabular} & & & 416 & & & 195 & 611 & \\
\hline $\begin{array}{l}\text { 2. Wendenschloß, } \\
\text { Krampenburg, } \\
\text { Müggelheim }\end{array}$ & \begin{tabular}{|lr} 
MB & 55 \\
SEG & 43 \\
SB/ & \\
KSB & 62 \\
GB & 7 \\
& \\
FB & 3 \\
BAD & 220 \\
Seebad \\
Wendens. \\
115 \\
\end{tabular} & $\begin{array}{l}\text { stark } \\
\text { mittel } \\
\text { gering } \\
\text { stark } \\
\text { stark } \\
\text { mittel } \\
\text { gering } \\
\end{array}$ & $\begin{array}{l}70 \\
30 \\
15 \\
70 \\
\\
51 \\
35\end{array}$ & $\begin{array}{|ll|}\text { Wand. } & 21 \\
\text { Radf. } & 35 \\
\text { ruhender } & \\
\text { Verkehr } & 250 \\
\text { Durch. } & \\
\text { Verkehr } & 0 \\
\text { Zeltplätze } & 3 \\
\end{array}$ & $\begin{array}{l}\text { gering } \\
\text { gering } \\
\text { mittel } \\
\text { nicht relevant } \\
\text { stark }\end{array}$ & $\begin{array}{l}5 \\
5 \\
40 \\
0 \\
80\end{array}$ & & $\begin{array}{l}\text { Bekanntes Naherholungsgebiet mit geringem bis mittleren Be- } \\
\text { sucherverkehr ( Zeltplatze und Wendenschloß-Marienlust) } \\
\text { Mittlere Belastung durch ruhenden Verkehr im Ortsbereich Wen- } \\
\text { denschloB und Parkplatz Marienlust/Mügelturm, Seebad Wen- } \\
\text { denschloB und freie Badestellen vorhanden. Das Bad war nur im } \\
\text { August und September geöffnet. Rückgang der Besucher } 1996: \\
1995 \text { auf nahezu } 1 / 5 \text { ( } 5.750: 25.800 \text { ), Zelttlatzze Krampenburg } \\
\text { und Kuhle Wampe mit Uferbelastung durch Stegbau und freie } \\
\text { Badestellen }\end{array}$ \\
\hline Punkte gesamt & & & 291 & & & 130 & 421 & \\
\hline $\begin{array}{l}\text { 3.Schmóckwitzer Br. } \\
\text { Rauchfangswerder } \\
\text { Zeuthener See }\end{array}$ & $\begin{array}{lr}\text { MB } & 125 \\
\text { SEG } & 9 \\
\text { SB/ } & \\
\text { KSB } & 58 \\
\text { GB } & 4 \\
& \\
\text { FB } & 4 \\
\text { BAD } & 400 \\
\text { BAD } & \\
\text { Eichw. } \\
100 \\
\end{array}$ & \begin{tabular}{|l} 
sehr stark \\
nicht relevant \\
gering \\
stark \\
stark \\
mittel \\
gering \\
\end{tabular} & $\begin{array}{l}90 \\
0 \\
15 \\
51 \\
51 \\
45\end{array}$ & \begin{tabular}{ll|} 
Wand. & 25 \\
Radf. & 15 \\
ruhender & \\
Verkehr 603 \\
Durchg. \\
Verkehr 166 \\
Zeltplätze 2 \\
Anlieger
\end{tabular} & $\begin{array}{l}\text { gering } \\
\text { gering } \\
\text { sehr stark } \\
\text { sehr stark } \\
\text { stark } \\
\text { mittel }\end{array}$ & $\begin{array}{l}5 \\
5 \\
85 \\
80 \\
75 \\
50\end{array}$ & & $\begin{array}{l}\text { Langjähriges Campingrevier und Naherholungsgebiet mit freien } \\
\text { Badestellen in Nähe Schmöckwitzer Brücke. Hier Konzentration } \\
\text { von starkem Durchgangs- und ruhendem Verkehr, Ufer- Bela- } \\
\text { stung durch Stegbau, zahlreiche Gaststätten nahe Schmöckwit- } \\
\text { zer Brücke und relativ günstige Parkmöglichkeiten in Seenăhe, } \\
\text { nach Suden geringer belastet } \\
\text { Brandenburger Ufer bebaut, Belastunmg durch Anlieger und } \\
\text { eine freie Badestelle }\end{array}$ \\
\hline Punkte gesamt & & & 267 & & & 300 & 567 & \\
\hline 4.Seddinsee & \begin{tabular}{|lr} 
MB & 76 \\
SEG & 94 \\
SB/ & \\
KSB & 16 \\
GB & 7 \\
& \\
FB & 5 \\
BAD & 30 \\
\end{tabular} & \begin{tabular}{|l|} 
stark \\
stark \\
nicht relevant \\
stark \\
stark \\
nicht relevant \\
\end{tabular} & \begin{tabular}{|l|}
75 \\
60 \\
0 \\
70 \\
60 \\
0 \\
\end{tabular} & 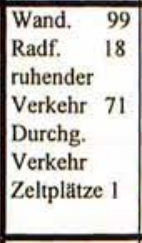 & $\begin{array}{l}\text { gering } \\
\text { gering } \\
\text { gering } \\
\text { mittel } \\
\text { mittel }\end{array}$ & $\begin{array}{l}15 \\
5 \\
25 \\
30 \\
30\end{array}$ & & $\begin{array}{l}\text { Bevorzugtes Motorboot- und Segelrevier, geringe Zugănglichkeit } \\
\text { durch sumpfige Ufer im nördlichern Seebereich (Naturschutzge- } \\
\text { biet), im suddlichen Seebereich Zugang nur aber Fußgänger- } \\
\text { brucke Wernsdorf, kleine Badestellen zwischen Oder-Spree- } \\
\text { Kanal und Zwiebusch, Zeltplatz auf der Insel Seddinwall }\end{array}$ \\
\hline Punkte gesamt & & & 265 & & & 105 & 370 & \\
\hline 5.Nördliche Dahme & $\begin{array}{lr}\text { MB } & 158 \\
\text { SEG } & 37 \\
\text { SB/ } & \\
\text { KSB } & 52 \\
\text { GB } & 8 \\
& \\
\text { FB } & 8 \\
\text { BAD } & 6 \\
\text { FluBbad } \\
\text { 400 } \\
\end{array}$ & \begin{tabular}{|l|} 
sehr stark \\
mittel \\
gering \\
stark \\
stark \\
nicht relevant \\
mittel \\
\end{tabular} & \begin{tabular}{|l|}
95 \\
30 \\
15 \\
75 \\
75 \\
0 \\
45 \\
\end{tabular} & $\begin{array}{|ll|}\text { Wand. } & 0 \\
\text { Radf. } & 0 \\
\text { ruhender } & \\
\text { Verkehr } & \\
\text { Durchg. } & \\
\text { Verkehr } & \\
\text { Zeltplatze } & \\
\text { Anlieger } & \\
\end{array}$ & \begin{tabular}{|l} 
nicht relevant \\
nicht relevant \\
mittel \\
sehr stark \\
nicht relevant \\
stark
\end{tabular} & $\begin{array}{l}0 \\
0 \\
45 \\
90 \\
0 \\
75\end{array}$ & & $\begin{array}{l}\text { Für die Offentlichkeit kaum zugănglicher Uferbereich, beidersei- } \\
\text { tig bebaute Ufer mit Wohn-, Gewerbe- und Industriegebieten } \\
\text { Starker Durchgangsverkehr uber Damm- und Lange Brücke, } \\
\text { uber Grünauer- und Wendenschloßstr. } \\
\text { Ein Flußbad Gartenstr. mit einer Belastung bis } 500 \text { Besucher } \\
\text { täglich. }\end{array}$ \\
\hline Punkte gesamt & & & 335 & & & 210 & 545 & \\
\hline
\end{tabular}

Legende: Keine relevante Umweltbelastung 0

$\begin{array}{lll}\text { geringe Umweltbelastung } & 1 & 25 \\ \text { mittlere Umweltbelastung } & 26 & 50 \\ \text { starke Umweltbelastung } & 51 & 75 \\ \text { sehr starke Umweltbelastung } & 76 & 100\end{array}$

Tabelle 5: Belastungsmatrix (nach Höchstwerten/h)

Verkehr sowohl in Richtung Müggelsee wie auch in Richtung Dahme bewegen kann und die höchste wasserseitige Belastung im Untersuchungsgebiet gemessen wurde. Zu beachten ist, daß diese Höchstbelastungen, wie in der vorderen Tabelle dargestellt, vordringlich an Hochsommertagen, an Wochenenden und feiertags sowie in der Zeit von 14.00 - 18.00 Uhr gemessen wurden.

Landseitig sind besonders die Uferbereiche im Grünauer Erholungsgebiet von der Regattastrecke bis Karolinenhof durch die Regattastrecke als nationale und internationale Wettkampfstrecke, durch Badende, Wanderer 
und Radfahrer stark belastet. Auf der Wendenschloßseite des Langen Sees treten starke Belastungen im Bereich der Zeltplätze Krampenburg und Kuhle Wampe sowie mittlere Belastungen von Wendenschloß über Schmetterlingshorst bis Marienlust ( und weiter zum Müggelturm) auf. Im Bereich der Schmöckwitzer Brükke sind der ruhende Verkehr und Durchgangsverkehr sehr stark belastend und die Badenden und die Zeltplätze stark belastend.

Aus den Analyseergebnissen wurden für den Auftraggeber konkrete Handlungsempfehlungen abgeleitet und übergeben.

\section{Literaturverzeichnis}

1. BVG:

Nahverkehrsatlas Berlin/Potsdam 1996/1997

2. DNR e.V.:

Wassersport und Naturschutz

Bonn 1981

3. DVWK (Hrsg.):

Gestaltung und ökologische Entwicklung von Seen in: Schriftenreihe des Deutschen Verbandes für Wasserwirtschaft und Kulturbau e. V., Heft 95 , Berlin 1991

4. Entwurf - Kurzfassung des Konzepts zur Entwicklung der Regattastrecke/Wassersportzentrum Grünau

Senatsverwaltung für Schule, Jugend und Sport

5. Eberlei, B.

Ökologische Tragfähigkeit räumlicher Nutzung Theoretische und praktische Erörterung am Modellbeispiel Steinhuder Meer.

Vom Fachbereich Landschaftspflege der Universität Hannover genehmigte Dissertation, 1985

6. Erbgut, W.

Umweltverträgliche Freizeitanlagen - Eine Anleitung zur Prüfung von Projekten des Ski-, Wasserund Golfsports aus der Sicht der Umwelt, Band II, Rechtsfragen, Berlin 1987, UBA -Berichte 6/87

7. Fremdenverkehrsverband Dahmeland, Fremdenverkehrsverein Köpenick e. V.:

Modellprojekt für den Fremdenverkehr im Land Brandenburg - NATOUREN - Wanderungen durch die Dahme-Seenlandschaft, 1995

8. Gareis-Grabmann, F-J.:

Landschaftsbild und Umweltverträglichkeit

Erich Schmidt Verlag, Berlin 1993

9. Hahn, P.:

Analyse des Konflikts - Umwelt/Sport aus landschaftsplanerischer Sicht,

Schriftenreihe Landschaftsentwicklung der TU Berlin, Nr. 65, Berlin 1989
10. Hofmeister, B.:

Berlin - Beiträge zu Geographie eines Großstadtraumes, Festschrift zum 45. Deutschen Geographentag in Berlin, vom 30.09.1985 - 02.10.1985, Dietrich Reimer Verlag, Berlin 1986

11. Initiative zum Wassersport Brandenburg Region "Suid-Ostberliner Wald- und Seengebiet Oder Spree“, Landkreis Oder - Spree

12. Krähe, Ch.(Hrsg.):

Wassersport auf Binnengewässern und Bodensee, Muiller Verlag, Heidelberg 1987

13. Kühl, H.:

Röhricht und Uferschutzmaßnahmen an den Berliner Spree- und Havelgewässern

Abschlußbericht 1991, Teil B: Zustand der Ufer und Röhrichtbestände des Seddinsees, des Langen Sees, der Großen Krampe und der Dahme

14. Lamey, H.-U., Richter, W.:

Naherholung in München - ein Modell

In: Grünstift - Forum, 1990

15. Landessportbund Berlin e.V.: Wassersportplan 1991

16. Regionalverband Bodensee - Oberschwaben Bodenseeuferplan nach der Genehmigung von 15. Nov. 1985

Ravensburg 1985

17. Schemel, H.J.:

Umweltverträgliche Freizeitanlagen - Eine Anleitung zur Prüfung von Projekten, des Ski-, Wasserund Golfsports aus der Sicht der Umwelt Band I, Analyse und Bewertung, Berlin 1987, UBABerichte 5/1987

18. Senatsverwaltung für Stadtentwicklung und Umweltschutz III:

Landschaftsprogramm, Berlin 1995

Erholung und Freiraumnutzung, Biotop und Artenschutz, Landschafts- Naturhaushalt/Umweltschutz

19. Senatsverwaltung für Stadtentwicklung, Umweltschutz und Technologie: Freiraumerholung in Berlin, Berlin 1995

20. Senatsverwaltung fuir Stadtentwicklung und Umweltschutz, Bezirksamt Köpenick: Grundlagen für die Bereichsentwicklungsplanung (BEP-Grundlagen) Bezirk Köpenick (Berlin 1991)

21. Täubert, U.:

Die Bedeutung der Projektbewertung für die Planung in der wasserwirtschaftlichen Praxis, in: Schriftenreihe des Deutschen Verbandes für Wasserwirtschaft und Kulturbau e.V., Heft 66, Berlin 1984 
22. UVP-Förderverein (Hrsg.):

Umweltvorsorge für ein Fluß-Ökosystem

UVP - Spezial 6, Dortmund 1993

23. Der Wassersportentwicklungsplan des Landes Brandenburg, Grundsätze und Ziele Potsdam 1996

24. Uhlemann, H.-J.

Berlin und die Märkischen Wasserstraßen, DSV-Verlag, Hamburg 1994

25. Wilde, H.:

Von den Camps in Köpenick, in: Grïnstift - Forum 1990

26. Wolff, F:

Gewässerfuihrer Berlin, Ed. Maritim

Hamburg 1993

27. Wolle, J.:

„Möge uns ein gnädiges Schicksal bewahren“ iiber die Regionalplanung und den Umgang mit Menschen

in: Grünstift - Forum 1990

\section{Fußnoten}

1) Beide Bereiche gehören zum Langen See. Um die unterschiedlichen landseitigen Belastungen des Südund Nordufers einschätzen zu können, werden sie aus methodischen Gründen getrennt.

\section{Verfasser}

Prof. Dr. sc. oec. Heinz Kroske

Fachbereich Ingenieurwesen/Wirtschaftsingenieurwesen Tel.: 03375/508327

Dr. oec. Bernd Lehne

Fachbereich Wirtschaft, Verwaltung und Recht

Tel.: 03375/508918

Technische Fachhochschule Wildau

Bahnhofstr. 1

15745 Wildau 